\title{
An eHealth System for Pressure Ulcer Risk Assessment Based on Accelerometer and Pressure Data
}

\author{
Dieter Hayn, ${ }^{1}$ Markus Falgenhauer, ${ }^{1}$ Jürgen Morak, ${ }^{1}$ Karin Wipfler, ${ }^{2}$ Viktoria Willner, ${ }^{3}$ \\ Walter Liebhart, ${ }^{4}$ and Günter Schreier ${ }^{1}$ \\ ${ }^{1}$ Safety and Security Department, AIT Austrian Institute of Technology GmbH, Reininghausstr 20, 8020 Graz, Austria \\ ${ }^{2}$ Hilfswerk Österreich, Apollog 4/5, 1070 Vienna, Austria \\ ${ }^{3}$ Salzburg Research Forschungsgesellschaft mbH, Jakob-Haringerstraße 5/3, 5020 Salzburg, Austria \\ ${ }^{4}$ ilogs mobile software GmbH, Krone Platz 1I, 9020 Klagenfurt, Austria \\ Correspondence should be addressed to Dieter Hayn; dieter.hayn@ait.ac.at
}

Received 20 October 2014; Revised 7 January 2015; Accepted 21 January 2015

Academic Editor: Josep Samitier

Copyright (C) 2015 Dieter Hayn et al. This is an open access article distributed under the Creative Commons Attribution License, which permits unrestricted use, distribution, and reproduction in any medium, provided the original work is properly cited.

\begin{abstract}
Pressure ulcers are a common skin disease which is associated with pain, reduced autonomy, social isolation, and reduced quality of life. There are several systems for monitoring of pressure ulcer-related risk factors on the market, but up to now no satisfactory solution is available, especially for people with medium pressure ulcer risk. We present a novel pressure ulcer risk assessment and prevention system, which combines the advantages of accelerometer and pressure sensors for monitoring pressure ulcer risk factors. Sensors are used for detection of repositionings of the person lying on the mattress. Sensor data are sent to a tablet where they are analysed and presented graphically. The system was evaluated in a long-term test at the homes of people of the target group. Results indicate that the system is able to detect movements of persons while lying in bed. Weak correlation in between mobility and Braden pressure ulcer risk was found (correlation factor $=0.31$ ). From our data, long-term trends could be visualized as well as $24 \mathrm{~h}$ mobility profiles. Such graphical illustrations might be helpful for caregivers in order to optimize care of people with medium to high pressure ulcer risk.
\end{abstract}

\section{Introduction}

Pressure Ulcers: General Description and Risk Factors. Pressure ulcers are a skin disease which is caused by mechanically induced ischemia. Pressure ulcers are associated with pain, reduced autonomy, social isolation, and reduced quality of life, leading to enormous costs for the health care system. In Germany in 2005 12.5\% of all patients within clinics and $6.6 \%$ in nursing homes have been affected by pressure ulcers. Studies in the Netherlands in 2005 even showed $16.9 \%$ in clinic, $25.5 \%$ for nursing homes, and $12.6 \%$ for home care patients [1]. A recent review of pressure ulcer prevalence and incidence across Scandinavia, Iceland, and Ireland showed prevalence rates of 8.9 to $25 \%$. Mean incidence varied in between 1.8 and $20 \%$ [2]. The total risk of an individual person to develop a pressure ulcer can be estimated by different scales (e.g., Braden scale), which are based on several risk factors, such as low activity, reduced mobility, temperature and skin moisture (e.g., due to incontinence), nutrition and drinking behaviour, and additional diseases such as diabetes mellitus and reduced blood pressure [3]. Malnutrition and depleted lean body mass are a predictor of adverse events among the elderly and those at risk for pressure ulcers [4]. More details concerning pressure ulcer prevention and treatment can be found in recent guidelines [5].

Accordingly, the American pressure ulcer guideline recommends twelve preventive activities: implementation of measures to reduce pressure ulcer risk; no high gradients of the head of the bed (prevention of shear-forces); regular repositioning; special attention in case of sitting positioning; heal protection by complete offload; support surfaces, pressure distributing devices and surfaces for high-risk persons and in operating rooms; no use of foam rings, foam cutouts, or donut-type devices; skin protectant for individuals with frequent fecal incontinence or double urinary and fecal incontinence; sufficient nutrition and fluid intake and patient education [6]. 
State-of-the-Art Pressure Ulcer Risk Monitoring. There are different types of special active or passive pressure ulcer mattresses. Passive mattresses are made of materials (foam, gel, air-filled, etc.) which adapt to the geometry of their load and therefore distribute the weight to a larger surface. Active mattresses usually contain various air chambers whose pressure is automatically varied over time. Although such mattresses are widely used and significantly reduce the pressure ulcer risk, they have three major disadvantages: (a) they have high costs and (b) noise during inflation and deflation which can be annoying and disturbing [7] and (c) they have negative effects on patient mobility and independence [7]. Therefore, they are usually used for patients at very high risk, while there is a lack of low cost prevention systems for patients with medium risk.

Other approaches for pressure ulcer prevention focus on detection of long-term pressure to particular skin regions. The most obvious type of detector is a pressure sensor, since pressure is the ultimate reason for pressure ulcers. There are several groups worldwide, who are currently researching the use of pressure sensor mats that can detect long-term pressure to particular skin regions [8]. Smith and Cooper [9] proposed a system consisting of various sensors, such as cameras, pressure sensors, and accelerometers. Sensors described in this patent could be pressure sensitive mats, optical sensors, weight sensing pads underneath each leg of the bed, and vertical accelerometers mounted to the bed's springs. Murakami et al. developed novel capacitive force sensors for measurement of skin interface force [10]. Zimlichman et al. used piezoelectric sensors mounted underneath the mattress to detect bed movements and activities [11]. Chenu et al. described a textile sensor that mounted on a wheelchair, consisting of three layers and based on the piezoresistive effect [12].

All such systems have several disadvantages. One is their high costs. Another crucial point is the location of the sensor system in the bed, which can be underneath or above the mattress. Though sensors underneath the mattress are less obtrusive, they are sensible rather to the location of the bed's slatted frame than to the monitored person. On the other hand, location of the sensor mat above the mattress leads to reduced air circulation, vulnerability in terms of fluids, and reduced comfort. Therefore, pressure based sensor mats are currently used for other applications like individual adaption of wheel chairs [13], but up to now, apart from laboratory settings, their application for pressure ulcer prevention is limited so far.

There are other approaches, monitoring the number of movements of the patient while lying in bed instead of pressure on discrete skin regions. This number closely correlates with long-term pressure exposure. Motion monitors are used for sleep stage tracking and can be useful for pressure ulcer risk estimation as well [14]. Sensors can be optical sensors, accelerometers, acoustic sensors, and so forth. Usually, sensors are mounted on the bed (e.g., $[15,16])$ or the patient himself [17]. Alqassim et al. [18] describe the use of a mobile phone's accelerometer for sleep monitoring. Hoque et al. [19] attached wireless 3D accelerometers to a mattress in order to monitor sleeping behaviour. However, existing

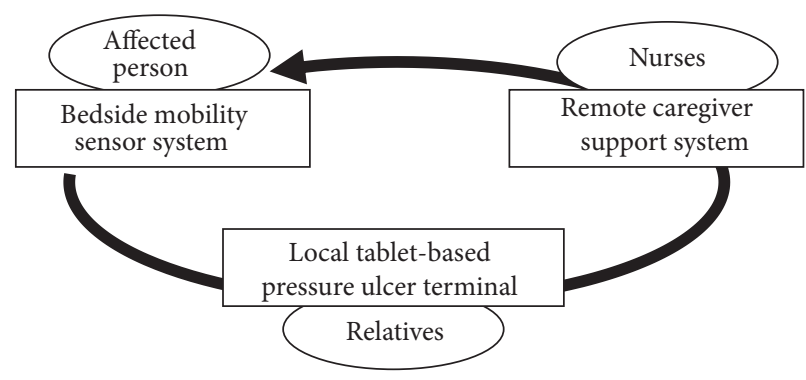

FIGURE 1: Overview of primary user interface for different stakeholders.

approaches only have sensors underneath the mattress (e.g., [20]), since sensors positioned right underneath the patient can reduce comfort and breathability. In conclusion, none of the existing products for pressure ulcer risk prevention fulfils the following basic requirements:

(i) sufficient reliability,

(ii) unobtrusiveness,

(iii) long-term usage,

(iv) low costs,

(v) easy mounting at various types of beds.

Additionally, feasibility of such a sensor system can only be validated by testing it for long times, at the patient's home, addressing people of the target group (elderly or disabled people with need of care). Nevertheless, no longterm analyses of usage of such a system in a home care scenario are available so far.

It was the aim of the present study to develop a pressure ulcer risk assessment system based on accelerometer and pressure data which is able to fulfil the requirements listed above. Since the rate of repositioning while lying in bed is closely related to the pressure on single skin regions [14] and since repositioning sensors are far easier to implement in a suitable way for continuous care, we decided not to detect the direct cause of pressure ulcers, that is, long-term pressure to single skin regions, but only one single reason for this direct cause, namely, the number of repositionings during lying in bed. The system's feasibility should be evaluated in a longterm test at the homes of people of the target group.

\section{Methods}

2.1. Overall Concept. A system for monitoring pressure ulcer risk factors in home care scenarios has been developed, consisting of a beside mobility sensor system, a local tabletbased pressure ulcer terminal, and a remote caregiver support system, providing access for affected persons, relatives, and nurses (see Figure 1).

2.2. User Centered Design. We used a user centered design approach for developing the system for monitoring pressure ulcer risk factors in a mobile home care scenario. Initially, from literature review and expert discussions we identified various types of sensors with potentially high benefit in 
pressure ulcer risk monitoring, including pressure sensor mats, weight and pressure based barycenter measurement, moisture sensors, accelerometers, cameras, and infrared sensors. During a stakeholder workshop with several experts the most promising approaches were selected [21]. These sensors have then been implemented and a first demonstrator was developed. The demonstrator was again presented to stakeholders, leading to a set of recommended adaption and optimization steps [22]. The resulting system will be described in the following. Finally, a prototypical system has been developed, whose feasibility has been evaluated in a study with people who were clients of the caregiver company in our project team (see Section 3).

2.3. Pressure Ulcer Risk Screening. A special Android software module for acquiring the pressure ulcer risk of an individual person has been developed, based on the Braden scale. The module was integrated in a preexisting mobile software solution for mobile home care. It was the purpose of the module to (a) enhance the preexisting software solution during routine home care and (b) to support acquisition of base line data for the feasibility study as described in Section 2.5. 41 items were recorded, including 24 administrative items (e.g., ID, name, and address), 6 items required for the Braden scale, and 11 study specific items (e.g., study group and informed consent signed). One of the project partners was a caregiver offering mobile home care in Austria. Nurses of the project's caregiver, who were already familiar with the preexisting software solution for mobile home care, were trained on usage of the new software module by members of the project team. Thereafter, the module was used in our feasibility study for pressure ulcer risk screening of the caregiver's clients.

2.4. Pressure Ulcer Monitoring System. An integrated system for monitoring of pressure ulcer risk factors in a mobile home care scenario has been developed. The system consisted of three major components, which will be described in detail in the following sections:

(1) accelerometer and pressure based motion detection,

(2) data collection and preprocessing unit,

(3) home terminal and user interface.

Figure 2 provides an overview of the complete sensor system.

2.4.1. Accelerometer and Pressure Based Motion Detection. Motion detection was done by the use of five 3D accelerometer sensors (ADXL345, Analog Devices, Inc., Boston, USA) and four force sensing resistors (FSR-406, Interlink Electronics, Inc., Camarillo, USA). All sensors were fixed on the mattress with a specially developed belt system. The design of the belt system enabled placement of the sensors on the lower surface of the mattress as well as on the top face, without disturbing the person in the bed. To enable mounting of the belt system to mattresses of different size, a mechanism for adjusting and straining the belt was included at the bottom side of the mattress.

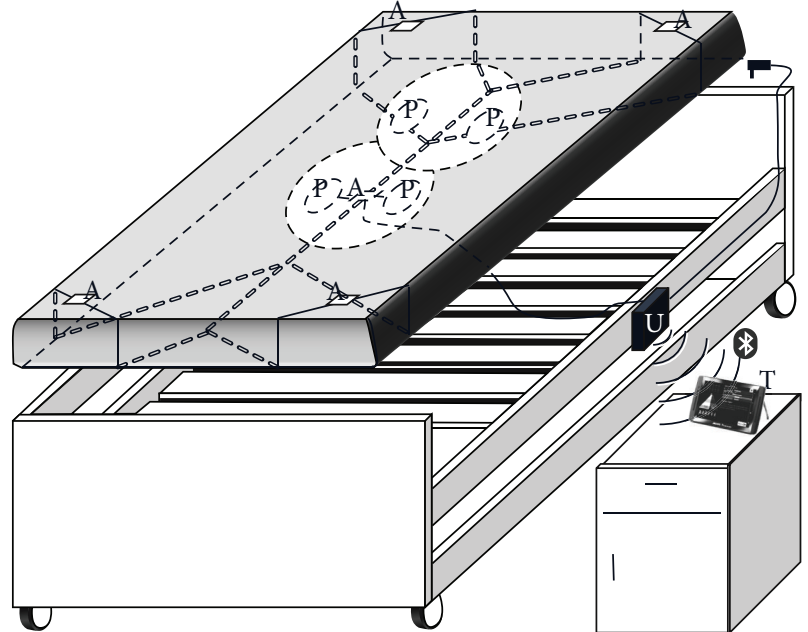

FIGURE 2: Overview of the sensor system, including accelerometers $(\mathrm{A})$, pressure sensors $(\mathrm{P})$, data collection and preprocessing unit $(\mathrm{U})$, and a tablet-based home terminal and user interface $(\mathrm{T})$.

One accelerometer sensor was located on the bottom side of the mattress in the middle of the bed between the mattress and the slatted frame. The other four sensors were located at the top face of the mattress, one in each corner. Since incontinence is common in high-risk pressure ulcer patients, special care was needed in terms of wetness when designing the system. Therefore, the sensors were isolated using shrinkable tubing. Low potential 3.3-volt power supply of the sensors was provided via cable by the data collection and preprocessing unit. Communication with the preprocessing unit was performed via serial peripheral interface (SPI). Force sensing resistors could resolute a weight of approximately $10 \mathrm{~g}$ to $10 \mathrm{~kg}$ and varied their resistance in a range of more than $1 \mathrm{M} \Omega$ to less than $3 \mathrm{k} \Omega$ and were used as pressure sensors, which were located slightly left and slightly right of the middle in the region of shoulders and hips.

The pressure sensors were also used for detecting whether a person was present within the bed or not.

2.4.2. Data Collection and Preprocessing Unit. Arduino Uno R3 and Arduino Mega 2560 R3 (Smart Projects, Italy, http://www.arduino.cc/) boards were used for collection and preprocessing of data from the sensors. Arduino is an open-source prototyping platform based on flexible, easyto-use hard- and software. Core of the Arduino board is an ATmega2560 processor (Atmel Corporation, San Jose, USA). The board allocates 54 digital IOs, 16 analog IOs, and an SPI interface. Universal asynchronous receiver transmitter (UART) was used for communicating with a Bluetooth attached to the board. A block diagram of the sensor electronic is shown in Figure 3.

All sensor data were filtered using a band pass filter of 0.9 to $2.6 \mathrm{~Hz}$. Thresholds for each direction $(x, y, z)$ of each sensor were defined. Thresholds could be adjusted to the type of mattress and to the weight of the person. Time and sensor ID were sent to the Android tablet whenever 


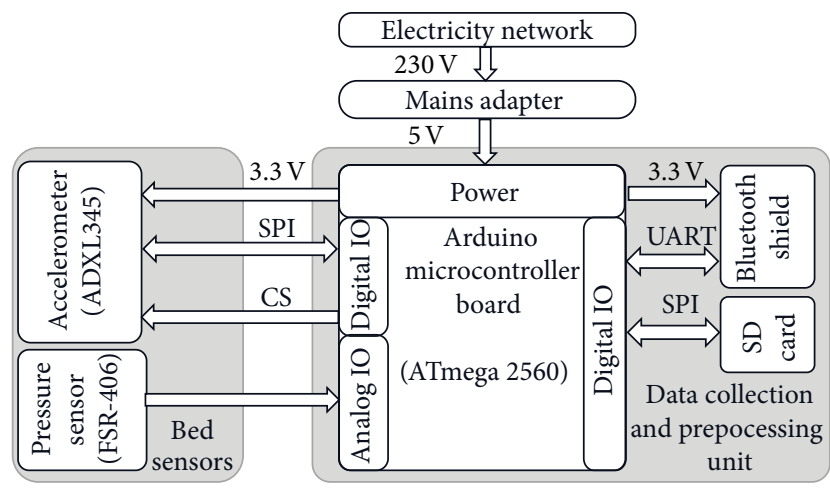

FIgURE 3: Block diagram of sensor hardware.

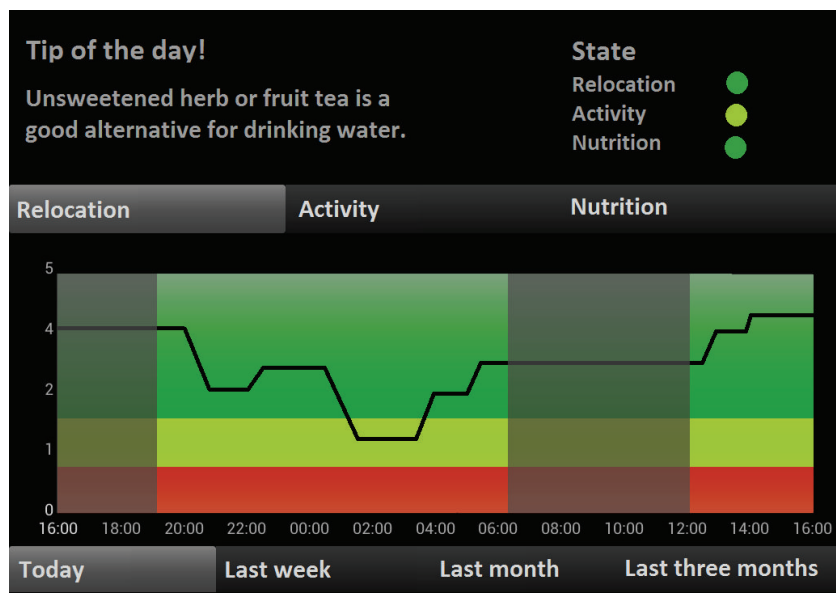

FIGURE 4: Screenshot of the main screen of the home terminal, showing the visualization of the collected sensor events. Grey areas indicate periods where the test person is absent.

a threshold was exceeded. Communication between Arduino board and home terminal was done via Bluetooth 2.1 + EDR and serial port profile (SPP) with a baud-rate of 115,200 bits/s. If no connection to the tablet was available, events were stored locally on an SD card and were transmitted after reestablishment of the connection.

2.4.3. Home Terminal and User Interface. An Archos80G9 Turbo (Archos Inc., Igny, France) mobile tablet device with Android 4.0.7 (Google Inc., Mountain View, USA) was used as home terminal and user interface. The home terminal (a) received and visualized the sensor data from the Arduino board and (b) enabled the user to manually input information about their physical activities, nutrition, and fluid intake on a daily base. The software was based on a home application developed in a preceding project, which focused on lifestyle management for elderly people [23].

The terminal analyzed and visualized all data and stored them in an SQLite database on the internal tablet storage. For data visualization, the events were aggregated in quarterly hour intervals. Figure 4 left shows the visualization of the collected sensor events that were displayed on the home terminal. The green, yellow, and red threshold areas provided information about the current pressure ulcer risk sensitively to the detected relocations. More details concerning the software are described in $[24,25]$.

2.5. Evaluation. Evaluation was done in a single centered, nonrandomized, nonblinded feasibility study which was conducted from July 2013 until February 2014 in the region of Salzburg, Austria.

Participants were recruited from the nurses of the project's caregiver. During their usual home care visits, specially trained nurses used our mobile pressure ulcer risk screening software to determine the individual pressure ulcer risk of their clients and asked if they were willing to participate in the study. Additionally, participators at antidecubitus courses held for informal carers were included.

In phase I of the study, the pressure ulcer risk of 200 persons should be determined. In phase II, 20 patients should be equipped with the pressure ulcer monitoring system.

2.6. Mobility Ration $R_{\text {active }}$. For long-term analysis of mobility data the whole study duration of each participant was divided in $15 \mathrm{~min}$ intervals. The number of active periods $n_{\text {active }}$ was defined as the number of quarterly hours, when (a) presence of the client in bed was detected and (b) movements were identified in at least 2 out of 4 quarterly hours right around the respective time point. Inactive periods $d_{\text {inactive }}$ summarized all 15 min intervals with 0 or 1 out of four quarterly hours with detected movements right around the respective time point (see Figure 5).

We calculated the mobility ratio $R_{\text {active }}$ of active periods as compared to active plus inactive periods, that is, the time period that the client was active in bed, on a daily basis:

$$
R_{\text {active }}=\frac{n_{\text {active }}}{n_{\text {inactive }}+n_{\text {active }}} .
$$

\section{Results}

The developed system has been evaluated in a feasibility study conducted in the region of Salzburg, Austria, from July 2013 until February 2014. During the screening phase I of the study, the pressure ulcer risk of 200 clients of the project's caregiver and of participators at the courses was determined. Initially, 19 clients agreed to participate in phase II of the feasibility study. However, five of these clients withdrew from the study prior to installation of the system after consultation of relatives or other persons of their trust. Two monitoring periods (starting in July 2013 and in December 2013) were conducted in phase II of the study.

3.1. Study Population. 14 persons were included in the monitoring phase of our feasibility study. The study was conducted in two subsequent periods (period 1 starting in July 2013 and period 2 starting in December 2013). One of the clients (male, Braden risk $=10$ due to severe physical disability) was $20 \mathrm{y}$ old. This client was the only patient taking part in both study phases on his own request. One client (male) was 41 . The age of the remaining 12 clients ( 3 male, 9 female) was $79 \pm 12 y$ (mean \pm std., range 56-93 y). 


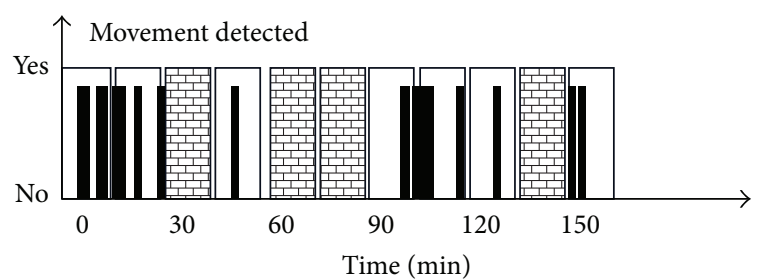

(a)

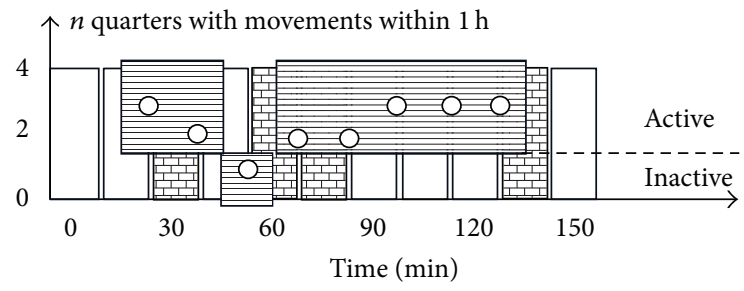

(b)

FIGURE 5: Identification of inactive time periods. (a) Black lines represent detected movements. White bars mark quarters with detected movements. Grey bars: quarters without detected movements. (b) White points represent the number of quarters with movements. Active period ( $\geq 2$ active quarters within $1 \mathrm{~h}$ ) and inactive period $(\leq 1$ active quarters) are highlighted.

Participants' median pressure ulcer risk according to Braden was 21.5 with an interquartile range of 16.3 to 23 . One client (age $93 \mathrm{y}$, female, Braden pressure ulcer risk =14) died of decrepitude after 21 monitoring days.

No relation in between the study and the client's death was identified. One client (age $90 \mathrm{y}$, female, Braden pressure ulcer risk $=17$ ) was withdrawn from the test by her caregivers due to a feeling of surveillance and subsequent worsening of her overall condition (increased blood pressure and insomnia). No data are available from this client. All other participants indicated no disturbance by the sensor system and no effect on sleep comfort.

In one case, no data could be collected due to technical problems. Study duration of the remaining clients was $57 \pm$ 39 days (mean \pm std., min 33 days, max 131 days). Detailed data of all participants are summarized in Table 1.

3.2. Monitoring Data Analysis. Different kinds and sizes of bed frames and mattresses were present at the patients' homes. By adapting the length of the belt system, mounting of the monitoring system was possible for each patient.

Altogether 8,111 hours of movement monitoring were recorded by our sensor system (mean \pm std. per patient $579 \pm 483 \mathrm{~h}$ ). An example of the data collected throughout the whole monitoring period is shown in Figure 6.

$24 \mathrm{~h}$ profiles of all patients are illustrated in Figure 7.

Table 2 summarizes the results as achieved for all patients. Analysis of factor $R_{\text {active }}$, representing the ratio of active periods as described above, revealed $0.28 \pm 0.12$ (mean \pm std.) per patient. The lowest value (0.11) was found for patient $13(\mathrm{~m}, 41 \mathrm{y}$, Braden pressure ulcer risk $=23)$ whereas the highest value (0.45) was found for patient 3 (f, 74 y, Braden
TABLE 1: Study population.

(a)

\begin{tabular}{lc}
\hline & Number \\
\hline$n$ patients & 197 \\
$n$ male/female & $71 / 126$ \\
$n$ with diabetes mellitus type I/II & $31 / 5$ \\
$n$ with other pressure ulcer-related diseases & 44 \\
$n$ with preexisting wound & 18 \\
$n$ using pressure ulcer prevention methods & 40 \\
\hline
\end{tabular}

(b)

\begin{tabular}{lcc}
\hline & Mn. \pm Std. & Range \\
\hline Age [y] & $78.7 \pm 13.3$ & {$[17-98]$} \\
Body weight $[\mathrm{kg}]^{*}$ & $73.5 \pm 17.0$ & {$[50-145]$} \\
Pressure ulcer risk (Braden) & $19 \pm 3.5$ & {$[9-23]$} \\
\hline
\end{tabular}

${ }^{*} 11$ data points $<50 \mathrm{~kg}$ have been filtered out.

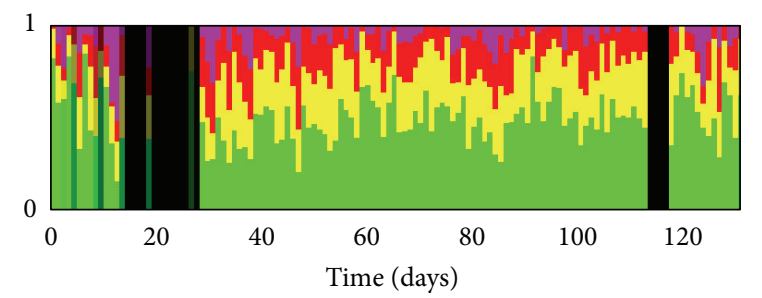

FIgURE 6: Mobility profile over the whole study period of client 4. For each day, the relative percentage of time intervals with a certain amount of inactive quarters within one hour is shown. Green: 0 or 1 inactive quarter per hour. Yellow: 2 inactive quarters per hour. Red: 3 inactive quarters per hour. Purple: 4 inactive quarters per hour. Days with little data are darkened.

pressure ulcer risk $=23$ ). Weak correlation in between $R$ and the clients' Braden pressure ulcer risk was found (correlation factor $=0.31$ ).

Detection of whether a person was present in bed or not was found to be highly unreliable, since trends in the pressure measured by the four pressure sensors appeared. This resulted in (a) missing data, when the person was present in bed but presence was not detected (no exact data are available concerning the frequency of this situation) and (b) undue data with very little movements detected, when presence was claimed though the person was not in bed. Accelerometer signals on the other hand proved to be stable over the monitoring period.

\section{Discussion}

A system for pressure ulcer risk assessment based on (a) accelerometer and pressure sensors and (b) manual input of pressure ulcer relevant data has been developed and its feasibility has been evaluated. The system is able to detect movements of persons while lying in bed. From these data, long-term trends can be visualized as well as $24 \mathrm{~h}$ mobility profiles. Such graphical illustrations might be helpful for 
TABLE 2: Monitoring data per patient. Age, pressure ulcer risk according to Braden, monitoring period, ratio of active time in bed $R_{\text {active}}$, number of manually documented nutrition, and activity items as well as a comment are shown.

\begin{tabular}{|c|c|c|c|c|c|c|c|c|}
\hline ID & Sex & Age [y] & $\begin{array}{l}\text { Pressure } \\
\text { ulcer risk }\end{array}$ & $\begin{array}{l}\text { Monitoring } \\
\text { period }[\mathrm{d}]\end{array}$ & $\begin{array}{l}\text { Measuring } \\
\text { period }[\mathrm{h}]\end{array}$ & $R_{\text {active }}$ & $\begin{array}{l}n \text { manual items/d } \\
\text { [nutrition/activity] }\end{array}$ & Comment \\
\hline 01 & M & 79 & 23 & 105 & 941 & 0.11 & $0.3 / 0.2$ & \\
\hline 02 & W & 73 & 23 & 53 & 691 & 0.32 & $0.1 / 0$ & \\
\hline 03 & $\mathrm{~W}$ & 74 & 23 & 84 & 692 & 0.45 & $0.2 / 0.1$ & Abnormal belt pos. \\
\hline 04 & W & 56 & 11 & 131 & 1586 & 0.25 & $0 / 0$ & \\
\hline 05 & $\mathrm{~W}$ & 62 & 22 & 98 & 1173 & 0.21 & $0.1 / 0.1$ & \\
\hline 06 & M & 20 & 10 & 75 & 29 & 0.40 & $11.0 / 10.3$ & Phases 1 and 2 \\
\hline 07 & $\mathrm{~W}$ & 90 & 17 & 0 & 0293 & N.A. & $0 / 0$ & Surveillance \\
\hline 08 & $\mathrm{~W}$ & 93 & 14 & 21 & 157 & 0.43 & $0 / 0$ & Deceased \\
\hline 09 & $\mathrm{~W}$ & 91 & 23 & 56 & 469 & 0.32 & $0 / 0$ & \\
\hline 10 & M & 82 & 21 & 33 & 403 & 0.12 & $0 / 0$ & \\
\hline 11 & $\mathrm{~W}$ & 81 & 19 & 0 & 0 & N.A. & $0 / 0$ & Techn. problems \\
\hline 12 & M & 84 & 18 & 41 & 566 & 0.30 & $0 / 0$ & \\
\hline 13 & M & 41 & 23 & 56 & 1087 & 0.11 & $0 / 0$ & \\
\hline 14 & $\mathrm{~W}$ & 90 & 22 & 43 & 53 & 0.39 & $0.2 / 0.1$ & \\
\hline Mn. & N.A. & 73 & 19 & 57 & 579 & 0.28 & $0.90 / 0.83$ & N.A. \\
\hline Std. & N.A. & 21 & 5 & 39 & 483 & 0.12 & $3.08 / 2.97$ & N.A. \\
\hline
\end{tabular}

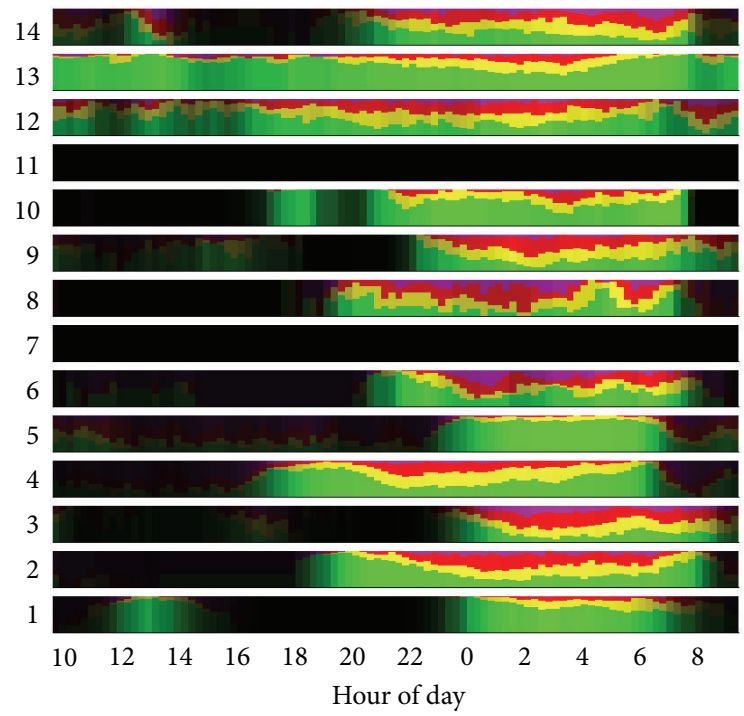

FIGURE 7: $24 \mathrm{~h}$ mobility profiles of all 14 clients. For each hour of day, the relative percentage of days with a certain amount of inactive quarters is shown. Green: 0 or 1 inactive quarter per hour. Yellow: 2 inactive quarters per hour. Red: 3 inactive quarters per hour. Purple: 4 inactive quarters per hour. Time points with little data are darkened.

caregivers in order to optimize care of people with medium pressure ulcer risk.

As compared to other approaches, our system has the advantage that (a) it can be mounted on every mattress; (b) it can be mounted on beds with every kind of frame and duckboard; (c) it has no physical effect on the sleep comfort; and (d) it can still detect movements in the head as well as in the foot region of the bed. In a next step, we are planning to completely avoid pressure measurements, especially for presence detection, in order to increase long-term stability of the system.

Pressure ulcers are caused by long-term static pressure to single skin regions. At the beginning of our project was our aim to detect situations where such long pressure applications to, for example, heel or head, are present. Such pressure sensor mats are also focus of most ongoing research activities of other groups. However, we found that low cost, easyto-use, reliable, and significant sensors based on pressure monitoring are hard to realize. Therefore, we decided not to detect the direct cause of pressure ulcers, that is, long-term pressure to single skin regions, but only one single reason for this direct cause, namely, the number of repositionings during lying in bed. Since the repositioning-rate is closely related to the pressure on single skin regions and since repositioning sensors are far easier to implement in a suitable way for continuous care, we intentionally accepted an expected reduction in sensitivity of our sensor system.

Manual data entry was hardly used by most of the patients (see Table 2). This was due to the high age of the patients and technical problems with the patient terminal. Six of the 14 patients never entered any data manually. Therefore, data concerning manual data entry must be interpreted with care. For future studies, more intuitive and usable solutions for manual data entry are suggested, such as radio frequency identification (RFID) or near field communication (NFC). 


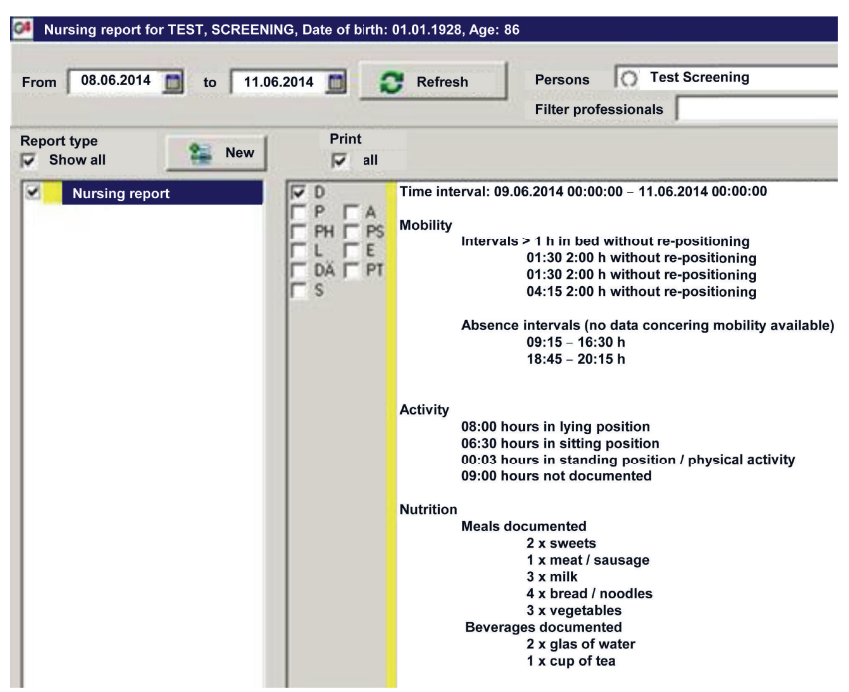

FIGURE 8: Example of a care report containing decubitus risk-related data as provided by our system.

Long-term analyses of such a technical system at the home of people of the system's target group are not easyto-perform. In the initial phase of the feasibility study, 200 clients of the project's care provider were screened for their pressure ulcer risk. 19 clients agreed to participate in the trial and 14 of those actually started with the study. Unfortunately, we do not have exact numbers concerning reasons for denial to participate. Nevertheless, from interviews with our study nurses we qualitatively identified some major concerns, such as concerns related to operating a tablet app; concerns related to electrical devices in the bed; concerns related to electromagnetic radiation (UMTS, Bluetooth); and concerns related to being under surveillance, especially in bed, including sexual habits. Special attention should be laid on these concerns during further investigation of our approach.

As a future step we are planning to extend nursing reports as currently used by several care providers, which was intended to include aggregated pressure ulcer risk-related information in a clearly arranged way. This way, the affected person, their relatives and nurses of a supporting caregiver should be involved in the care process, as illustrated in Figure 1. Therefore, additionally to the sensor data, nutrition, drinking behaviour, and physical activity can be collected on a tablet-based home terminal. These data will be transmitted to the caregiver's backend software system and integrated as one additional block in the preexisting reports, which are generated on a daily basis by the backend system. This workflow has been tested in an offline setting retrospectively using data of one participant from one single data. The resulting section in the online report is shown in Figure 8.

\section{Conclusion}

Monitoring of mobility in bed is an important step in pressure ulcer prevention. Mounting accelerometers on a belt which is clamped on the mattress proved to be a promising approach for unobtrusive movement detection, since it combines the advantages of accelerometer based approaches with pressure measurement underneath the mattress. Long-term trends and $24 \mathrm{~h}$ mobility plots can be generated and may be useful for caregivers. Future work will focus on more reliable methods for presence detection, completely avoiding (unstable) pressure measurement.

\section{Conflict of Interests}

The authors declare no conflict of interests.

\section{Authors' Contribution}

Dieter Hayn was project leader of the WUDOKU project. He supervised the development and evaluation process. Additionally, he was responsible for biosignal processing algorithms and for analysis of the sensor and other study data and for statistical analyses. Markus Falgenhauer, together with Jürgen Morak, developed the sensor system. Additionally he was responsible for the tablet software and for the electronic data capture system used during the study. Jürgen Morak was responsible for development of the sensor system. Karin Wipfler was responsible for recruitment of and contact to the test persons, including achievement of the screening data. She was also strongly involved in the design of the test system and the study protocol. Viktoria Willner was responsible for study evaluation and she was strongly involved in the study design. Walter Liebhart was responsible for user centered design and for development of the mobile electronic data capture system for screening data achievement. Additionally, he was responsible for inclusion of the study data into the caregiver's electronic documentation system. Günter Schreier was responsible for scientific supervision of the whole project and especially the evaluation process.

\section{Acknowledgment}

This project was partly funded by the Austrian Research Promotion Agency (FFG) under Grant no. 830161.

\section{References}

[1] R. J. G. Halfens, T. Dassen, and A. Tannen, "Prävalenz von Dekubitus," in Manual der Wundheilung, pp. 179-185, 2007.

[2] Z. Moore, E. Johanssen, and M. van Etten, "A review of PU prevalence and incidence across Scandinavia, Iceland and Ireland (part I)," Journal of Wound Care, vol. 22, no. 7, pp. 361368, 2013.

[3] B. J. Braden and N. Bergstrom, "The braden scale for predicting pressure sore risk," Nursing Research, vol. 36, no. 4, pp. 205-210, 1987.

[4] M. D. Litchford, B. Dorner, and M. E. Posthauer, "Malnutrition as a precursor of pressure ulcers," Advances in Wound Care (New Rochelle), vol. 3, no. 1, pp. 54-63, 2014.

[5] National Pressure Ulcer Advisory Panel, European Pressure Ulcer Advisory Panel, and Pan Pacific Pressure Injury Alliance, Prevention and Treatment of Pressure Ulcers: Quick Reference 
Guide, edited by E. Haesler, Cambridge Media, Perth, Australia, 2014.

[6] Wound, Ostomy, and Continence Nurses Society (WOCN), Guideline for Prevention and Management of Pressure Ulcers, WOCN Clinical Practice Guideline no. 2, Wound, Ostomy and Continence Nurses Society (WOCN), Mount Laurel, NJ, USA, 2010.

[7] Wounds International, International Review. Pressure Ulcer Prevention: Pressure, Shear, Friction and Microclimate in Context. A Consensus Document, Wounds International, London, UK, 2010.

[8] M. Verbunt and C. Bartneck, "Sensing senses: tactile feedback for the prevention of decubitus ulcers," Applied Psychophysiology Biofeedback, vol. 35, no. 3, pp. 243-250, 2010.

[9] T. E. Smith and C. L. Cooper, "Apparatus and method for reducing the risk of decubitus ulcers," US patent Nr. US 6646 $556 \mathrm{~B} 1,2003$.

[10] C. Murakami, Y. Ishikuro, and M. Takahashi, "Feasibility of novel four degrees of freedom capacitive force sensor for skin interface force," BioMedical Engineering Online, vol. 11, article 90, 2012.

[11] E. Zimlichman, Z. Shinar, R. Rozenblum et al., "Using continuous motion monitoring technology to determine patient's risk for development of pressure ulcers," Journal of Patient Safety, vol. 7, no. 4, pp. 181-184, 2011.

[12] O. Chenu, N. Vuillerme, M. Bucki, B. Diot, F. Cannard, and Y. Payan, "TexiCare: an innovative embedded device for pressure ulcer prevention. Preliminary results with a paraplegic volunteer," Journal of Tissue Viability, vol. 22, no. 3, pp. 83-90, 2013.

[13] M. Ferguson-Pell and M. D. Cardi, "Prototype development and comparative evaluation of wheelchair pressure mapping system," Assistive Technology, vol. 5, no. 2, pp. 78-91, 1993.

[14] A. N. Exton-Smith and R. W. Sherwin, "The prevention of pressure sores significance of spontaneous bodily movements," The Lancet, vol. 2, no. 7212, pp. 1124-1126, 1961.

[15] J. M. Kortelainen, M. O. Mendez, A. M. Bianchi, M. Matteucci, and S. Cerutti, "Sleep staging based on signals acquired through bed sensor," IEEE Transactions on Information Technology in Biomedicine, vol. 14, no. 3, pp. 776-785, 2010.

[16] B. H. Jansen and K. Shankar, "Sleep staging with movementrelated signals," International Journal of Bio-Medical Computing, vol. 32, no. 3-4, pp. 289-297, 1993.

[17] G. Aigner, G. Schreier, and M. Daumer, "Mobile Akzelerometrie zur Messung der Lage in Ruhe und Schlaf," in Tagungsband der eHealth 2011, D. Hayn and E. Ammenwerth, Eds., pp. 181-187, Österreichische Computer Gesellschaft, Wien, Austria, 2011.

[18] S. Alqassim, M. Ganesh, S. Khoja, M. Zaidi, F. Aloul, and A. Sagahyroon, "Sleep apnea monitoring using mobile phones," in Proceedings of the IEEE 14th International Conference on eHealth Networking, Applications and Services (Healthcom '12), pp. 443-446, Beijing, China, October 2012.

[19] E. Hoque, R. F. Dickerson, and J. A. Stankovic, "Monitoring body positions and movements during sleep using WISPs," in Proceedings of the Wireless Health (WH '10), pp. 44-53, San Diego, Calif, USA, October 2010.

[20] J. Paalasmaa, M. Waris, H. Toivonen, L. Leppakorpi, and M. Partinen, "Unobtrusive online monitoring of sleep at home," in Proceedings of the 34th Annual International Conference of the IEEE Engineering in Medicine and Biology Society (EMBS '12), pp. 3784-3788, San Diego, Calif, USA, September 2012.
[21] A. Zaunmayr, D. Hayn, A. Dohr et al., "Evaluation of sensor concepts for optimized decubitus prophylaxis and therapy," in Proceedings of the eHealth Conference, G. Schreier, D. Hayn, A. Hörbst, and E. Ammenwerth, Eds., pp. 157-163, OCG, Vienna, Austria, 2012.

[22] S. Zöscher, Development of a mobile patient terminal for the sensor based decubitus prophylaxis for slow go- and no-go empowerment [M.S. thesis], FH Joanneum, 2013.

[23] M. Drobics, A. Dohr, S. Giesecke, and G. Schreier, "LiKeITRFID-based KeepInTouch lifestyle monitoring," in Handbook of Ambient Assisted Living-Technology for Healthcare, Rehabilitation and Well-Being, J. C. Augusto, M. Huch, A. Kameas et al., Eds., vol. 11 of Ambient Intelligence and Smart Environments, 2012.

[24] M. Falgenhauer, S. Zöscher, J. Morak et al., "A patient centered system for decubitus prevention based on nutrition, drinking, physical activity and sleep monitoring," Studies in Health Technology and Informatics, vol. 192, p. 1033, 2013.

[25] M. Falgenhauer, S. Zöscher, J. Morak et al., "A patient centered sensor system for decubitus risk predection and prevention," Biomedizinische Technik, vol. 58, supplement 1, p. 4245, 2013. 

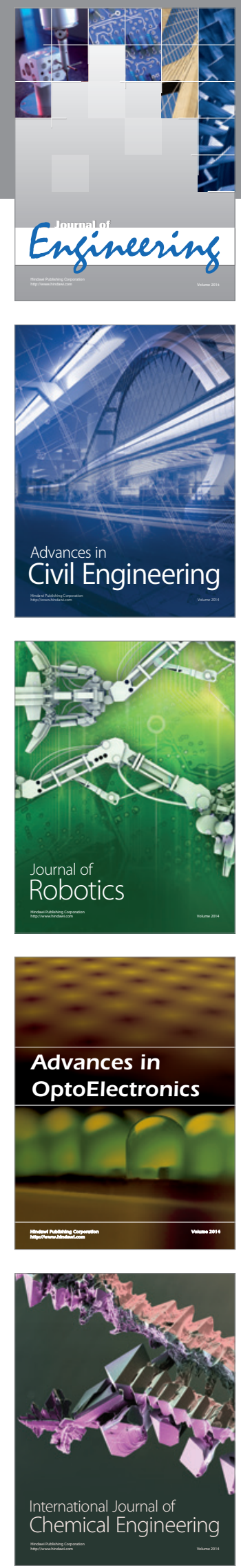

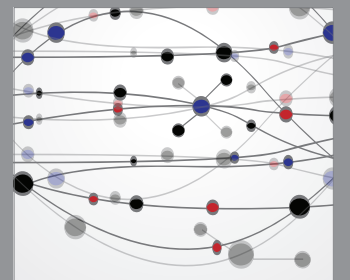

The Scientific World Journal
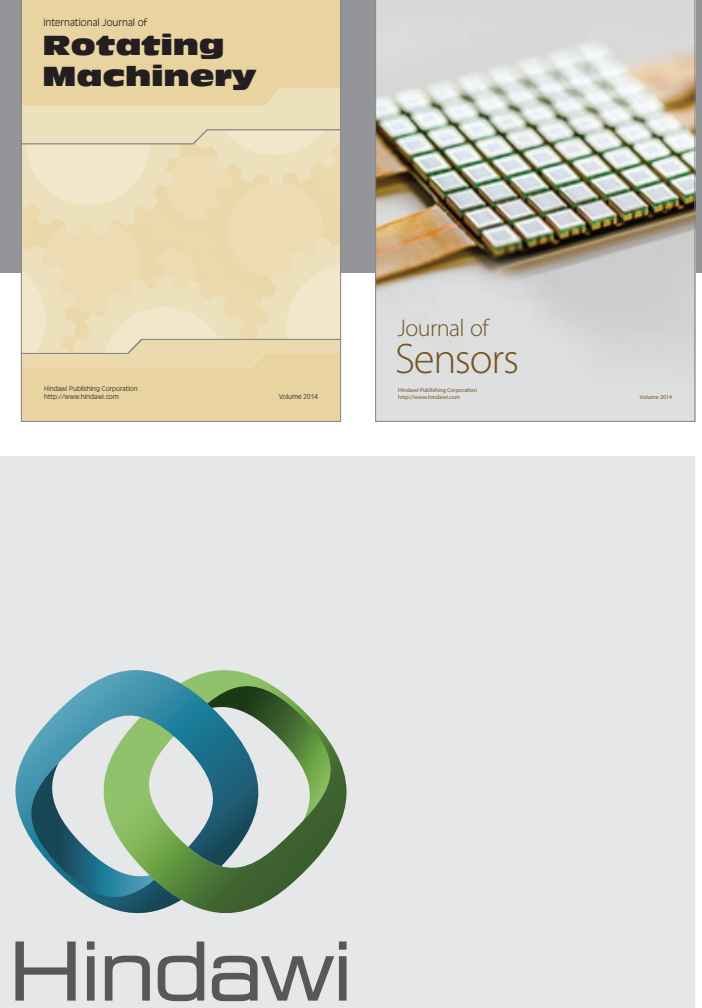

Submit your manuscripts at http://www.hindawi.com
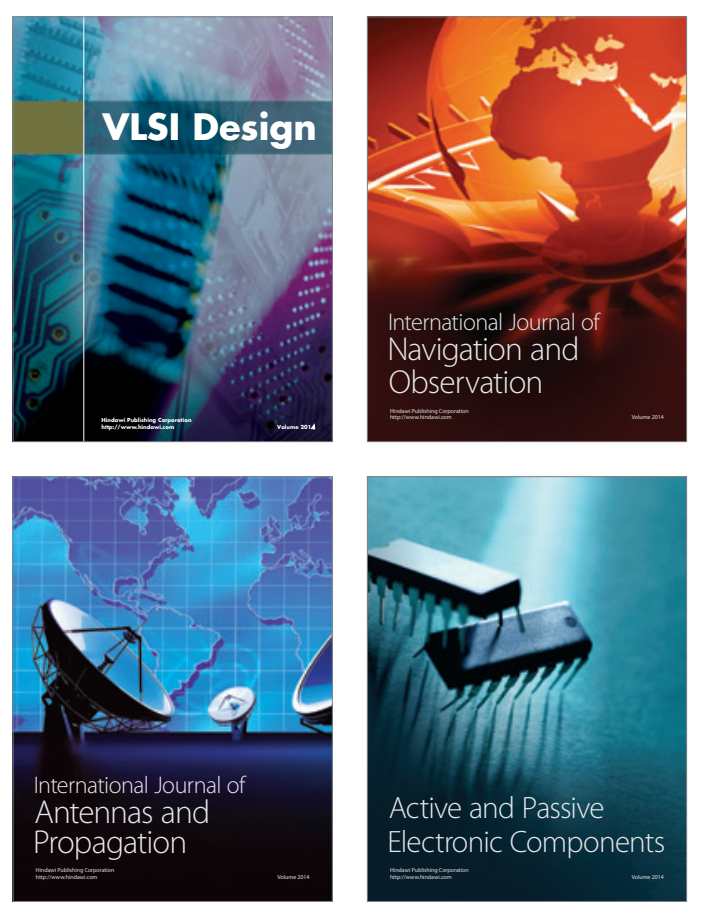
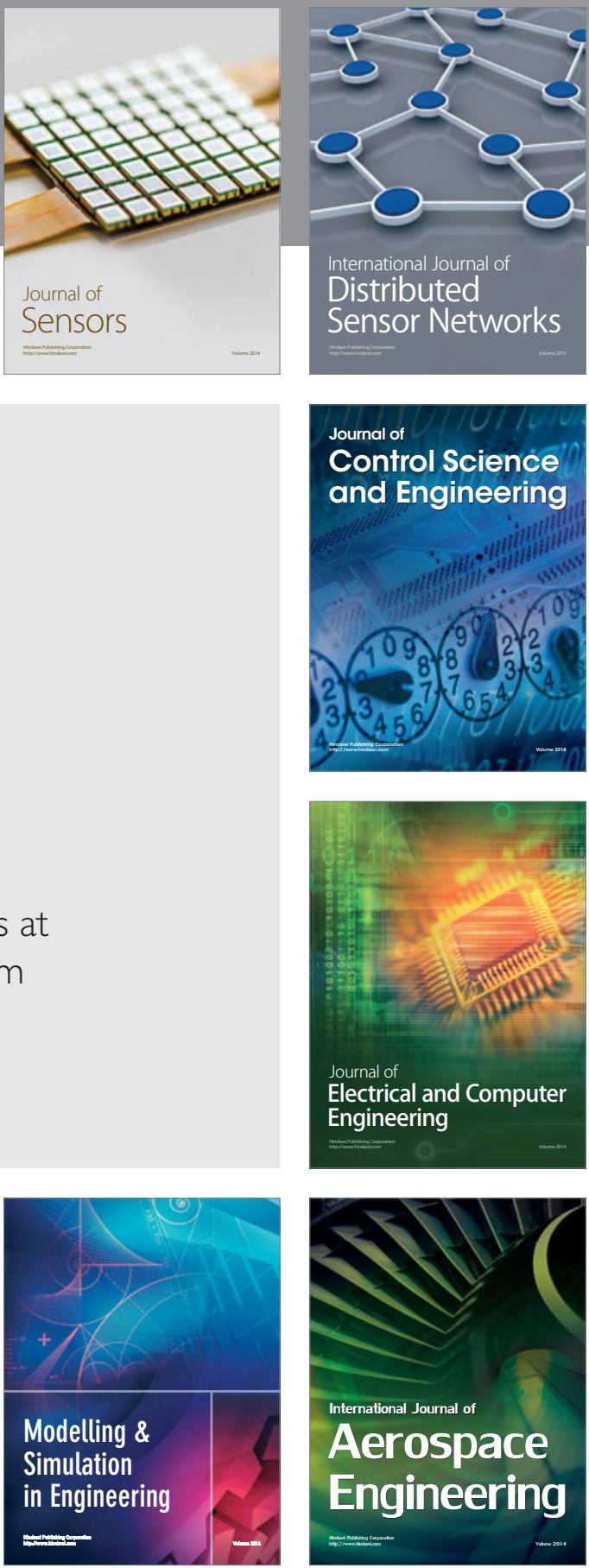

Journal of

Control Science

and Engineering
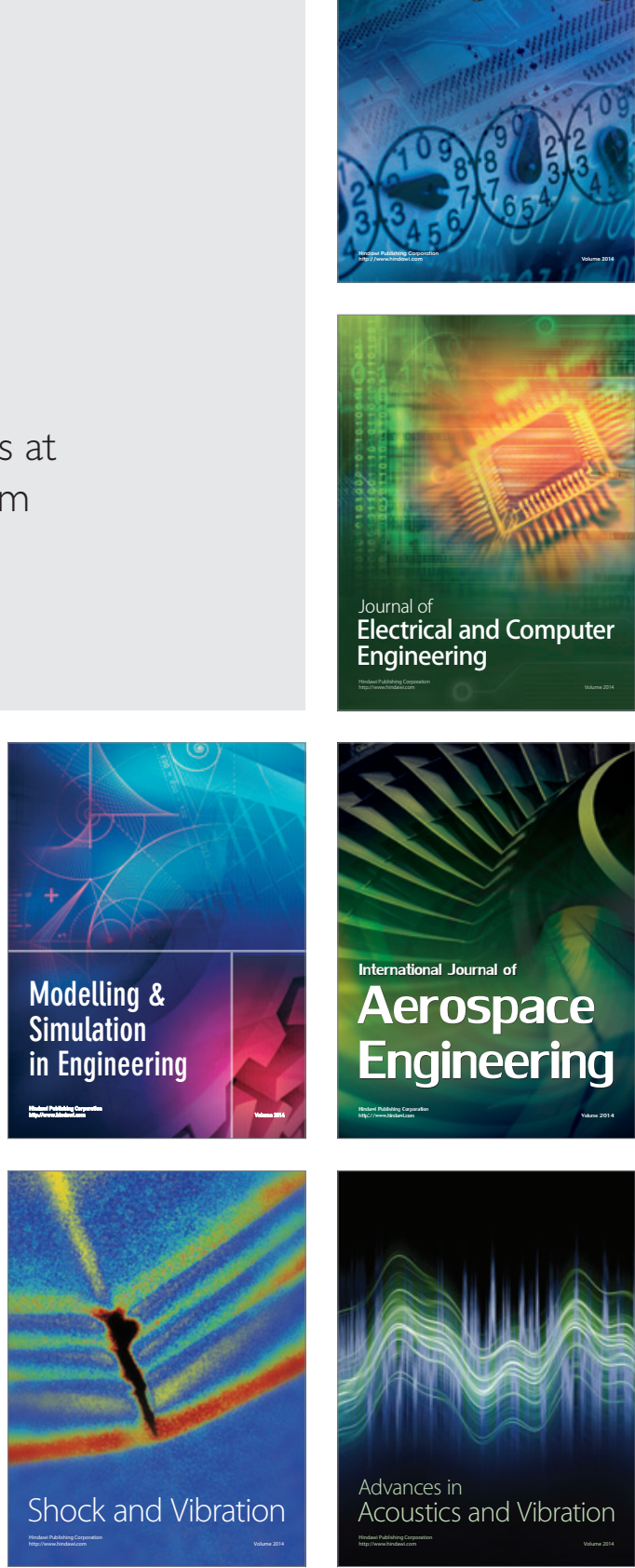